\title{
Existence and nonexistence of positive solutions for nonlinear higher order BVP with fractional integral boundary conditions in Banach spaces
}

Fulya Yoruk Deren ${ }^{*}$

"Correspondence:

fulya.yoruk@ege.edu.tr Department of Mathematics, Ege University, Bornova, Izmir, 35100, Turkey

\begin{abstract}
By means of the fixed point theory for a strict set contraction operator, this paper investigates the existence, nonexistence, and multiplicity of positive solutions for a nonlinear higher order boundary value problem with four point fractional integral boundary conditions in Banach spaces. In addition, an example is worked out to illustrate the main results.
\end{abstract}

MSC: 34B10; 39A10; 34B18; 45G10

Keywords: fixed point theorem; measure of noncompactness; positive solutions

\section{Introduction}

Let $(E,\|\cdot\|)$ be a real Banach space and $P \subset E$ be a cone of $E$. The goal of this paper is to study the existence, nonexistence, and multiplicity of positive solutions for the following higher order boundary value problem with fractional integral boundary conditions:

$$
\begin{aligned}
&{ }^{c} D^{\alpha} x(t)= \lambda_{1} a(t) f\left(t, x, x^{\prime}, \ldots, x^{(n-2)}\right) \\
&+\lambda_{2} b(t) g\left(t, x, x^{\prime}, \ldots, x^{(n-2)}, T x, S x\right), \quad t \in(0,1), \\
& x^{(i)}(0)=\theta, \quad 0 \leq i \leq n-3, \\
& x^{(n-2)}(0)+x^{(n-1)}(0)=I^{\delta} x^{(n-2)}(\eta), \\
& x^{(n-2)}(1)+x^{(n-1)}(1)+I^{\delta} x^{(n-2)}(\mu)=\theta,
\end{aligned}
$$

where $\theta$ is the zero element of $E, \lambda_{1}, \lambda_{2}$ are positive parameters, $n-1<\alpha<n(n \in \mathbb{N}$ and $n \geq 3),{ }^{c} D^{\alpha}$ is the Caputo fractional derivative of order $\alpha, \delta \geq 3, I^{\delta}$ is the Riemann-Liouville fractional integral of order $\delta, 1 / 4<\mu \leq \eta<3 / 4, f \in \mathcal{C}\left(J \times P^{n-1}, P\right), g \in \mathcal{C}\left(J \times P^{n+1}, P\right)(J=$ $[0,1])$, the coefficients $a, b \in \mathcal{C}\left((0,1), \mathbb{R}^{+}\right)$may be singular at $t=0$ or $t=1$. Here,

$$
T x(t)=\int_{0}^{t} K(t, s) x(s) d s, \quad S x(t)=\int_{0}^{1} H(t, s) x(s) d s,
$$

in which $K \in C\left[D, \mathbb{R}^{+}\right], D=\{(t, s) \in J \times J: t \geq s\}, H \in C\left[J \times, \mathbb{R}^{+}\right]$.

\section{Springer}

(c) 2015 Yoruk Deren; licensee Springer. This is an Open Access article distributed under the terms of the Creative Commons Attribution License (http://creativecommons.org/licenses/by/4.0), which permits unrestricted use, distribution, and reproduction in any medium, provided the original work is properly credited. 
Recently, there has been much attention on the fractional differential equations because of their applications in a variety of different areas of sciences including physics, chemistry, engineering etc. Thus, intensive study has been done to investigate the positive solutions for the nonlinear boundary value problems of fractional differential equations. For instance, in [1], Zhao et al. studied the following problem:

$$
\begin{aligned}
& D^{q} y(t)+r(t) f\left(y_{t}\right)=0, \quad \forall t \in(0,1), q \in(n-1, n], \\
& y^{(i)}(0)=\theta, \quad 0 \leq i \leq n-3, \\
& \alpha y^{(n-2)}(t)-\beta y^{(n-1)}(t)=\eta(t), \quad t \in[-\tau, 0], \\
& \gamma y^{(n-2)}(t)+\delta y^{(n-1)}(t)=\xi(t), \quad t \in[1,1+a] .
\end{aligned}
$$

Existence results of at least one or two positive solutions are established to the fractional functional differential equation by constructing a special cone and using the Krasnoselskii fixed point theorem.

In [2], Zhang considered the following boundary value problem for a fractional differential equation:

$$
\begin{aligned}
& D^{\alpha} u(t)+q(t) f\left(u, u^{\prime}, \ldots, u^{(n-2)}\right)=0, \quad t \in(0,1), \alpha \in(n-1, n], \\
& u(0)=u^{\prime}(0)=\cdots=u^{(n-2)}(0)=u^{(n-2)}(1)=0 .
\end{aligned}
$$

The author obtained the existence of positive solutions by using the fixed point theorem for mixed monotone operator. For more details and examples, we refer the reader to [3-10] and the references therein.

On the other hand, the existence results of positive solutions for integer order differential equations have been studied extensively by several researchers (see [11-16] and the references therein), but, as far as we know, only a few papers consider the BVP for higher order fractional differential equations in Banach spaces. (See $[7,17]$ and the references therein.) So, the aim of this paper is to fill this gap.

In this paper, we obtain the existence, multiplicity, and nonexistence of positive solutions for the BVP (1.1), (1.2) in Banach spaces. The argument is based upon the Kuratowski measure of noncompactness and fixed point theorem for strict set contraction operator. To our knowledge, the existence results, especially obtained for higher order fractional boundary value problems jointly with fractional integral boundary conditions are rarely seen when the nonlinear term takes values in an abstract space.

Let the real Banach space $E$ endowed with the norm $\|x\|$ be a partially ordered by a cone $P$ of $E$, i.e., $x \leq y$ if and only if $y-x \in P$. Recall that $P$ is said to be normal if there exists a constant $N>0$ such that $\theta \leq x \leq y$ implies $\|x\| \leq N\|y\|$. ( $N$ is called the normal constant of $P$.) In the present paper, we always assume that $P$ is normal in $E$ and without loss of generality, we suppose that the normal constant $N=1$.

The basic space using in this paper is $C[J, E]$. Clearly, $C[J, E]$ is a Banach space with the supremum norm $\|x\|_{c}=\sup \|x(t)\|$ and $Q=\{x \in C[J, E]: x(t) \geq \theta, t \in J\}$ is a cone of the Banach space $C[J, E]$.

A function $x \in C[J, E]$ whose $\alpha$ derivative exists on $J$ is called a solution of (1.1), (1.2) if $x$ obeys (1.1), (1.2). $x$ is a positive solution of (1.1), (1.2) if, in addition, $x(t) \geq \theta$ for $t \in(0,1)$ and $x(t) \not \equiv \theta$. 
For convenience of the reader, we first state some basic definitions and lemmas which can be found in $[4,5,18-21]$.

Definition 1.1 $([4,5])$ The Riemann-Liouville fractional integral of order $\alpha \in \mathbb{R}^{+}$for a continuous function $h:(0, \infty) \rightarrow \mathbb{R}$ is defined by

$$
I^{\alpha} h(t)=\frac{1}{\Gamma(\alpha)} \int_{0}^{t}(t-s)^{\alpha-1} h(s) d s
$$

where $\Gamma(\cdot)$ is the Euler Gamma function, provided that the integral exists.

Definition $1.2([4,5])$ If $h \in C^{n}[0,1]$, then the Caputo fractional derivative of order $\alpha$ is defined by

$$
{ }^{c} D^{\alpha} h(t)=\frac{1}{\Gamma(n-\alpha)} \int_{0}^{t}(t-s)^{n-\alpha-1} h^{(n)}(s) d s=I^{n-\alpha} h^{(n)}(t)
$$

where $n-1<\alpha<n, n=[\alpha]+1$ and $[\alpha]$ denotes the integer part of the real number $\alpha$.

Remark 1.1 ([4,5]) If $\alpha=n \in \mathbb{N}_{0}$, then the Caputo derivative coincides with a conventional $n$th order derivative of the function $h(t)$.

Lemma 1.1 ([4, 5]) If $\alpha>\beta>0$, then for $h(t) \in L(0,1)$, the equality

$$
\left({ }^{c} D^{\beta} I^{\alpha} h\right)(t)=I^{\alpha-\beta} h(t)
$$

is verified almost everywhere on $[0,1]$.

Lemma 1.2 $([4,5])$ Let $n=[\alpha]+1$ for $\alpha \notin \mathbb{N}$ and $n=\alpha$ for $\alpha \in \mathbb{N}$. If $y(t) \in C^{n}[0,1]$, then

$$
\left(I^{\alpha} D^{\alpha} y\right)(t)=y(t)-\sum_{i=0}^{n-1} \frac{y^{(i)}(0)}{i !} t^{i}
$$

Lemma $1.3([4,5])$ Let $\alpha>0$ and $n=[\alpha]+1$ for $\alpha \notin \mathbb{N}$ and $n=\alpha$ for $\alpha \in \mathbb{N}$. If $h(t) \in C[0,1]$, then the homogeneous fractional differential equation

$$
{ }^{c} D^{\alpha} h(t)=0
$$

has a solution

$$
h(t)=c_{1}+c_{2} t+c_{3} t^{2}+\cdots+c_{n} t^{n-1},
$$

where $c_{i} \in \mathbb{R}(i=1,2, \ldots, n)$.

Definition $1.3([18,19])$ Let $E$ be a real Banach space and $S$ be a bounded subset of $E$. Let $\alpha(S)=\inf \left\{\delta>0: S=\bigcup_{i=1}^{m} S_{i}\right.$ with $\left.\operatorname{diam}\left(S_{i}\right) \leq \delta, i=1,2, \ldots, m\right\}$. Then $\alpha(S)$ is called the Kuratowski measure of noncompactness. 
In this paper, we use $\alpha(\cdot)$ and $\alpha_{C}(\cdot)$ to denote the Kuratowski measure of noncompactness of a bounded set in $E$ and $C[J, E]$, respectively. For details of the definition and the properties of the measure of noncompactness, we refer the reader to [18-21] and the references therein.

Lemma 1.4 ([18]) If $H \subset C[I, E]$ is bounded and equicontinuous, then $\alpha(H(t))$ is continuous on $I$ and

$$
\alpha_{C}(H)=\max _{t \in I} \alpha(H(t)), \quad \alpha\left(\left\{\int_{I} x(t) d t: x \in H\right\}\right) \leq \int_{I} \alpha(H(t)) d t
$$

where $I=[a, b], H(t)=\{x(t): x \in H\}, t \in I$.

Definition 1.4 ([19]) Let $P$ be a cone of real Banach space $E$. If $P^{*}=\left\{\psi \in E^{*}: \psi(x) \geq\right.$ $0, \forall x \in P$ then $P^{*}$ is called a dual cone of cone $P$.

Throughout this paper, for any $y_{1}, y_{2}, \ldots, y_{n+1} \in P$ and $\psi \in P^{*}$ with $\|\psi\|=1$, we define

$$
\begin{aligned}
& f^{\beta}=\lim _{\sum_{i=1}^{n-1}\left\|y_{i}\right\| \rightarrow \beta} \sup \max _{t \in J} \frac{\left\|f\left(t, y_{1}, y_{2}, \ldots, y_{n-1}\right)\right\|}{\sum_{i=1}^{n-1}\left\|y_{i}\right\|}, \\
& g^{\beta}=\lim _{\sum_{i=1}^{n+1}\left\|y_{i}\right\| \rightarrow \beta} \sup \max _{t \in J} \frac{\left\|g\left(t, y_{1}, y_{2}, \ldots, y_{n+1}\right)\right\|}{\sum_{i=1}^{n+1}\left\|y_{i}\right\|}, \\
& (\psi f)_{\beta}=\lim _{\sum_{i=1}^{n-1}\left\|y_{i}\right\| \rightarrow \beta} \inf \min _{t \in J} \frac{\psi\left(f\left(t, y_{1}, y_{2}, \ldots, y_{n-1}\right)\right)}{\sum_{i=1}^{n-1}\left\|y_{i}\right\|}, \\
& (\psi g)_{\beta}=\lim _{\sum_{i=1}^{n+1}\left\|y_{i}\right\| \rightarrow \beta} \inf \min _{t \in J} \frac{\psi\left(g\left(t, y_{1}, y_{2}, \ldots, y_{n+1}\right)\right)}{\sum_{i=1}^{n+1}\left\|y_{i}\right\|},
\end{aligned}
$$

where $\beta$ is 0 or $\infty$.

Lemma 1.5 [19] Let $K$ be a cone in a Banach space $E$ and $K_{r, R}=\{x \in K: r \leq\|x\| \leq R\}$, $R>r>0$. Assume that $A: K_{r, R} \rightarrow K$ is a strict set contraction such that one of the following two conditions hold:

(i) $\|A x\| \geq\|x\|, \forall x \in K,\|x\|=r ;\|A x\| \leq\|x\|, \forall x \in K,\|x\|=R$.

(ii) $\|A x\| \leq\|x\|, \forall x \in K,\|x\|=r ;\|A x\| \geq\|x\|, \forall x \in K,\|x\|=R$.

Then $A$ has a fixed point $x \in K_{r, R}$ such that $r \leq\|x\| \leq R$.

\section{Several lemmas}

It is convenient to list the following assumptions which are to be used throughout the paper:

(H1) $f \in C\left(J \times P^{n-1}, P\right), g \in C\left(J \times P^{n+1}, P\right)$ and for any $r>0, f\left(t, u_{1}, u_{2}, \ldots, u_{n-1}\right)$, $g\left(t, u_{1}, u_{2}, \ldots, u_{n+1}\right)$ are uniformly continuous on $J \times P_{r}^{n-1}$ and $J \times P_{r}^{n-1} \times P_{k^{*} r} \times P_{h^{*} r}$, respectively. Here, $k^{*}, h^{*}, P_{r}$ are defined by

$$
\begin{aligned}
& k^{*}=\sup _{t \in[0,1]} \int_{0}^{1} K(t, s) d s, \quad h^{*}=\sup _{t \in[0,1]} \int_{0}^{1} H(t, s) d s, \\
& P_{r}=\{u \in P:\|u\| \leq r\} .
\end{aligned}
$$


(H2) $a, b \in C((0,1),[0, \infty))$ may be singular at $t=0$ or $t=1, a(t), b(t)$ do not vanish identically on any subinterval of $(0,1)$ with

$$
\int_{0}^{1}(a(s)+b(s)) m(s) d s<+\infty
$$

where $m(s)$ will be given in (2.30).

(H3) There exist nonnegative functions $L_{j}(\cdot), M_{k} \in L[0,1](j=1,2, \ldots, n-1 ; k=1,2, \ldots$, $n+1)$ such that

$$
\begin{aligned}
& \alpha\left(f\left(t, D_{1}, D_{2}, \ldots, D_{n-1}\right)\right) \leq \sum_{j=1}^{n-1} L_{j}(t) \alpha\left(D_{j}\right), \\
& \alpha\left(g\left(t, D_{1}, D_{2}, \ldots, D_{n+1}\right)\right) \leq \sum_{k=1}^{n+1} M_{k}(t) \alpha\left(D_{k}\right), \quad \forall t \in J .
\end{aligned}
$$

Here $D_{i} \subset P(i=1,2, \ldots, n+1)$ are bounded and

$$
\rho \int_{0}^{1} m(s)\left(\lambda_{1} a(s) L(s)+\lambda_{2} b(s) M(s)\right) d s<1,
$$

where

$$
\begin{aligned}
& L(s)=\sum_{j=1}^{n-2} \frac{L_{j}(s)}{(n-2-j) !}+L_{n-1}(s), \\
& M(s)=\sum_{k=1}^{n-2} \frac{M_{k}(s)}{(n-2-k) !}+M_{n-1}(s)+\frac{k^{*}}{(n-3) !} M_{n}(s)+\frac{h^{*}}{(n-3) !} M_{n+1}(s),
\end{aligned}
$$

and $k^{*}$ and $h^{*}$ are given by (2.1).

In order to obtain the existence and nonexistence of positive solutions, we will consider the following auxiliary problem:

$$
\begin{aligned}
&{ }^{c} D^{\alpha-n+2} y(t)= \lambda_{1} a(t) f\left(t, I^{n-2} y, \ldots, I^{1} y, y\right) \\
&+\lambda_{2} b(t) g\left(t, I^{n-2} y, \ldots, I^{1} y, y, T\left(I^{n-2} y\right), S\left(I^{n-2} y\right)\right), \quad t \in(0,1), \\
& y(0)+y^{\prime}(0)= I^{\delta} y(\eta) \\
& y(1)+y^{\prime}(1)+I^{\delta} y(\mu)=\theta
\end{aligned}
$$

where

$$
I^{j}(y)(t)=\frac{1}{\Gamma(j)} \int_{0}^{t}(t-s)^{j-1} y(s) d s \quad(j=1,2, \ldots, n-2) .
$$

Lemma 2.1 The higher order fractional boundary value problem (1.1), (1.2) has a solution if and only if the nonlinear fractional boundary value problem (2.4), (2.5) has a solution.

Proof Let $x$ be a solution of the higher order fractional boundary value problem (1.1), (1.2) and $y(t)={ }^{c} D^{n-2} x(t)$. Then from the boundary value conditions (1.2) and the definition of 
the Caputo fractional derivative, we obtain

$$
\begin{aligned}
& y(t)=D^{n-2} x(t)=x^{(n-2)}(t) \\
& I^{1} y(t)=I^{1} x^{(n-2)}(t)=\frac{1}{\Gamma(1)} \int_{0}^{t} x^{(n-2)}(s) d s=x^{(n-3)}(t), \\
& I^{2} y(t)=I^{2} x^{(n-2)}(t)=\frac{1}{\Gamma(2)} \int_{0}^{t}(t-s) x^{(n-2)}(s) d s=x^{(n-4)}(t), \\
& I^{n-2} y(t)=I^{n-2} x^{(n-2)}(t)=\frac{1}{\Gamma(n-2)} \int_{0}^{t}(t-s)^{n-3} x^{(n-2)}(s) d s=x(t), \\
& { }^{c} D^{\alpha-n+2} y(t)=\frac{1}{\Gamma(n-\alpha)} \int_{0}^{t}(t-s)^{n-\alpha-1} y^{\prime \prime}(s) d s \\
& =\frac{1}{\Gamma(n-\alpha)} \int_{0}^{t}(t-s)^{n-\alpha-1} x^{(n)}(s) d s \\
& ={ }^{c} D^{\alpha} x(t) \text {, }
\end{aligned}
$$

which imply that

$$
\begin{aligned}
& y(0)+y^{\prime}(0)=I^{\delta} y(\eta), \\
& y(1)+y^{\prime}(1)+I^{\delta} y(\mu)=\theta .
\end{aligned}
$$

Hence, $y(t)=x^{(n-2)}(t)$ is a solution of the fractional boundary value problem (2.4), (2.5).

Conversely, if $y$ is a solution of the fractional boundary value problem (2.4), (2.5), and letting $x(t)=I^{n-2} y(t)$, then it follows from the definition of the Caputo fractional derivative and the boundary value conditions (2.5) that

$$
\begin{aligned}
& x^{\prime}(t)=D^{1} I^{n-2} y(t)={ }^{c} D^{1} I^{1} I^{n-3} y(t)=I^{n-3} y(t), \\
& x^{\prime \prime}(t)=D^{2} x(t)={ }^{c} D^{2} I^{n-2} y(t)={ }^{c} D^{2} I^{2} I^{n-4} y(t)=I^{n-4} y(t), \\
& \vdots \\
& x^{(n-2)}(t)=D^{n-2} x(t)={ }^{c} D^{n-2} I^{n-2} y(t)=y(t), \\
& { }^{c} D^{\alpha} x(t)=I^{n-\alpha}\left(x^{(n)}\right)(t)=I^{n-\alpha}\left(I^{n-2} y\right){ }^{(n)}(t)=I^{n-\alpha}\left(y^{\prime \prime}\right)(t)={ }^{c} D^{\alpha-n+2} y(t),
\end{aligned}
$$

which indicate that

$$
\begin{aligned}
& x^{(i)}(0)=\theta, \quad 0 \leq i \leq n-3, \\
& x^{(n-2)}(0)+x^{(n-1)}(0)=I^{\delta} x^{(n-2)}(\eta), \\
& x^{(n-2)}(1)+x^{(n-1)}(1)+I^{\delta} x^{(n-2)}(\mu)=\theta .
\end{aligned}
$$

Finally, $x(t)=I^{n-2} y(t)$ is a solution of the higher order fractional boundary value problem (1.1), (1.2). Therefore, the proof of Lemma 2.1 is completed. 
Lemma 2.2 For any $h \in \mathcal{C}[(0,1), E]$ with $\int_{0}^{1}(1-s)^{\alpha-n} h(s) d s<+\infty$, the following fractional boundary value problem:

$$
\begin{aligned}
& { }^{c} D^{\alpha-n+2} y(t)=h(t), \quad t \in(0,1), \\
& y(0)+y^{\prime}(0)=\theta, \\
& y(1)+y^{\prime}(1)=\theta
\end{aligned}
$$

has a unique solution

$$
y(t)=\int_{0}^{1} G(t, s) h(s) d s
$$

where

$$
G(t, s)= \begin{cases}\frac{(1-s)^{\alpha-n+1}(1-t)+(t-s)^{\alpha-n+1}}{\Gamma(\alpha-n+2)}+\frac{(1-s)^{\alpha-n}(1-t)}{\Gamma(\alpha-n+1)}, & s \leq t \\ \frac{(1-s)^{\alpha-n+1}(1-t)}{\Gamma(\alpha-n+2)}+\frac{(1-s)^{\alpha-n}(1-t)}{\Gamma(\alpha-n+1)}, & t \leq s .\end{cases}
$$

Proof Let $y(t)$ be a solution of the boundary value problem (2.6), (2.7). Applying the operator $I^{\alpha-n+2}$ to both sides of (2.6), by Lemma 1.2, we reduce (2.6) to an equivalent integral equation

$$
y(t)=I^{\alpha-n+2} h(t)+c_{1}+c_{2} t .
$$

Thus, differentiating (2.10), we have

$$
y^{\prime}(t)=I^{\alpha-n+1} h(t)+c_{2} .
$$

By the boundary conditions of (2.7), we get

$$
\begin{aligned}
& c_{1}=I^{\alpha-n+2} h(1)+I^{\alpha-n+1} h(1), \\
& c_{2}=-I^{\alpha-n+2} h(1)-I^{\alpha-n+1} h(1) .
\end{aligned}
$$

Substituting these values into (2.10), we obtain

$$
\begin{aligned}
y(t)= & \frac{1}{\Gamma(\alpha-n+2)} \int_{0}^{t}(t-s)^{\alpha-n+1} h(s) d s+(1-t)\left[I^{\alpha-n+2} h(1)+I^{\alpha-n+1} h(1)\right] \\
= & \frac{1}{\Gamma(\alpha-n+2)} \int_{0}^{t}(t-s)^{\alpha-n+1} h(s) d s \\
& +\frac{1-t}{\Gamma(\alpha-n+2)} \int_{0}^{1}(1-s)^{\alpha-n+1} h(s) d s+\frac{1-t}{\Gamma(\alpha-n+1)} \int_{0}^{1}(1-s)^{\alpha-n} h(s) d s \\
= & \int_{0}^{1} G(t, s) h(s) d s .
\end{aligned}
$$

Therefore, the proof of Lemma 2.2 is completed. 
Lemma 2.3 For any $h \in \mathcal{C}[(0,1), E]$ with $\int_{0}^{1}(1-s)^{\alpha-n} h(s) d s<+\infty$, the following fractional boundary value problem:

$$
\begin{aligned}
& { }^{c} D^{\alpha-n+2} y(t)=h(t), \quad t \in(0,1), \\
& y(0)+y^{\prime}(0)=I^{\delta} y(\eta), \\
& y(1)+y^{\prime}(1)+I^{\delta} y(\mu)=\theta,
\end{aligned}
$$

has a unique solution

$$
y(t)=\int_{0}^{1} H(t, s) h(s) d s,
$$

where

$$
\begin{aligned}
& H(t, s)= G(t, s)+\frac{1}{\psi}\left[2\left(1+\frac{\mu^{\delta+1}}{\Gamma(\delta+2)}\right)-t\left(1+\frac{\mu^{\delta}}{\Gamma(\delta+1)}\right)\right] I^{\delta} G(\eta, s) \\
&+\frac{1}{\psi}\left[1-\frac{2 \eta^{\delta+1}}{\Gamma(\delta+2)}-t\left(1-\frac{\eta^{\delta}}{\Gamma(\delta+1)}\right)\right] I^{\delta} G(\mu, s), \quad t, s \in[0,1], \\
& \psi=2\left(1-\frac{\eta^{\delta}}{\Gamma(\delta+1)}\right)\left(1+\frac{\mu^{\delta+1}}{\Gamma(\delta+2)}\right)-\left(1+\frac{\mu^{\delta}}{\Gamma(\delta+1)}\right)\left(1-\frac{2 \eta^{\delta+1}}{\Gamma(\delta+2)}\right) .
\end{aligned}
$$

Here, $I^{\delta} G(\eta, s)$ and $I^{\delta} G(\mu, s)$ denote the Riemann-Liouville integral of $G(t, s)$ with respect to $t=\eta$ and $t=\mu$, respectively.

Proof Let

$$
u(t)=\int_{0}^{1} G(t, s) h(s) d s
$$

Then by Lemma $2.2, u(t)$ verifies

$$
\begin{aligned}
& { }^{c} D^{\alpha-n+2} u(t)=h(t), \quad t \in(0,1), \\
& u(0)+u^{\prime}(0)=\theta, \\
& u(1)+u^{\prime}(1)=\theta .
\end{aligned}
$$

Suppose that $y(t)$ is a solution of the boundary value problem (2.13), (2.14), and let

$$
z(t)=y(t)-u(t), \quad t \in[0,1],
$$

then $z(t)$ satisfies the following fractional boundary value problem:

$$
\begin{aligned}
& { }^{c} D^{\alpha-n+2} z(t)=\theta, \quad t \in(0,1), \\
& z(0)+z^{\prime}(0)=I^{\delta} z(\eta)+I^{\delta} u(\eta), \\
& z(1)+z^{\prime}(1)+I^{\delta} z(\mu)+I^{\delta} u(\mu)=\theta .
\end{aligned}
$$


Thus, we deduce from Lemma 1.3 that

$$
z(t)=c_{0}+c_{1} t, \quad t \in[0,1], c_{0}, c_{1} \in \mathbb{R} .
$$

Replacing $z(t)$ into (2.23), we get

$$
\begin{aligned}
& c_{0}=\frac{1}{\psi}\left[2 I^{\delta} u(\eta)\left(1+\frac{\mu^{\delta+1}}{\Gamma(\delta+2)}\right)+I^{\delta} u(\mu)\left(1-\frac{2 \eta^{\delta+1}}{\Gamma(\delta+2)}\right)\right], \\
& c_{1}=-\frac{1}{\psi}\left[I^{\delta} u(\mu)\left(1-\frac{\eta^{\delta}}{\Gamma(\delta+1)}\right)+I^{\delta} u(\eta)\left(1+\frac{\mu^{\delta}}{\Gamma(\delta+1)}\right)\right],
\end{aligned}
$$

where

$$
\psi=2\left(1-\frac{\eta^{\delta}}{\Gamma(\delta+1)}\right)\left(1+\frac{\mu^{\delta+1}}{\Gamma(\delta+2)}\right)-\left(1+\frac{\mu^{\delta}}{\Gamma(\delta+1)}\right)\left(1-\frac{2 \eta^{\delta+1}}{\Gamma(\delta+2)}\right)
$$

Finally, replacing (2.25) into (2.24), we have

$$
\begin{aligned}
z(t)= & \frac{1}{\psi}\left[2\left(1+\frac{\mu^{\delta+1}}{\Gamma(\delta+2)}\right)-t\left(1+\frac{\mu^{\delta}}{\Gamma(\delta+1)}\right)\right] I^{\delta} u(\eta) \\
& +\frac{1}{\psi}\left[1-\frac{2 \eta^{\delta+1}}{\Gamma(\delta+2)}-t\left(1-\frac{\eta^{\delta}}{\Gamma(\delta+1)}\right)\right] I^{\delta} u(\mu) .
\end{aligned}
$$

It follows from (2.21) and (2.27) that the integral equation (2.15) is satisfied. Therefore, the proof of Lemma 2.3 is completed.

Remark 2.1 Note that $\psi>0$ for $\frac{1}{4}<\mu \leq \eta<\frac{3}{4}$ and $\delta \geq 3$, since we have

$$
\begin{aligned}
\psi= & 2\left(1+\frac{\eta^{\delta}}{\Gamma(\delta+1)}\right)\left(1+\frac{\mu^{\delta+1}}{\Gamma(\delta+2)}\right)-\frac{4 \eta^{\delta}}{\Gamma(\delta+1)}\left(1+\frac{\mu^{\delta+1}}{\Gamma(\delta+2)}\right) \\
& -\left(1+\frac{\mu^{\delta}}{\Gamma(\delta+1)}\right)\left(1-\frac{2 \eta^{\delta+1}}{\Gamma(\delta+2)}\right) \\
\geq & \left(1+\frac{\eta^{\delta}}{\Gamma(\delta+1)}\right)\left(1+\frac{2 \mu^{\delta+1}}{\Gamma(\delta+2)}+\frac{2 \eta^{\delta+1}}{\Gamma(\delta+2)}\right)-\frac{4 \eta^{\delta}}{\Gamma(\delta+1)}\left(1+\frac{\mu^{\delta+1}}{\Gamma(\delta+2)}\right) \\
= & 1-\frac{3 \eta^{\delta}}{\Gamma(\delta+1)}-\frac{2 \eta^{\delta} \mu^{\delta+1}}{\Gamma(\delta+1) \Gamma(\delta+2)}+\frac{2 \eta^{\delta+1}}{\Gamma(\delta+2)}+\frac{2 \eta^{2 \delta+1}}{\Gamma(\delta+1) \Gamma(\delta+2)}+\frac{2 \mu^{\delta+1}}{\Gamma(\delta+2)} \\
\geq & 1-\frac{3 \eta^{\delta}}{\Gamma(\delta+1)}+\frac{2 \eta^{\delta+1}}{\Gamma(\delta+2)}+\frac{2 \mu^{\delta+1}}{\Gamma(\delta+2)} \\
> & \frac{2 \eta^{\delta+1}}{\Gamma(\delta+2)}+\frac{2 \mu^{\delta+1}}{\Gamma(\delta+2)} \\
> & 0 .
\end{aligned}
$$

Lemma 2.4 Let $n-1<\alpha<n$. Then $G(t, s)$ given by the expression (2.9) has the following properties:

(i) $G(t, s) \in \mathcal{C}([0,1] \times[0,1)), G(t, s)>0, t, s \in(0,1)$. 
(ii) There exists a positive function $\varphi \in \mathcal{C}(0,1)$ such that

$$
G(t, s) \leq m(s), \quad t \in[0,1], s \in(0,1)
$$

and

$$
G(t, s) \geq \varphi(s) m(s), \quad t \in\left[\frac{1}{4}, \frac{3}{4}\right], s \in(0,1),
$$

where

$$
m(s)=\frac{2(1-s)^{\alpha-n+1}}{\Gamma(\alpha-n+2)}+\frac{(1-s)^{\alpha-n}}{\Gamma(\alpha-n+1)}, \quad s \in[0,1) .
$$

Proof From the definition of $G(t, s)$, it is easy to see that (i) holds. Now, we will prove the inequalities of the Green's function $G(t, s)$.

Let us define the functions $G_{1}(t, s)$ and $G_{2}(t, s)$ as follows:

$$
\begin{aligned}
& G_{1}(t, s)=\frac{(1-s)^{\alpha-n+1}(1-t)+(t-s)^{\alpha-n+1}}{\Gamma(\alpha-n+2)}+\frac{(1-s)^{\alpha-n}(1-t)}{\Gamma(\alpha-n+1)}, \quad s \leq t ; \\
& G_{2}(t, s)=\frac{(1-s)^{\alpha-n+1}(1-t)}{\Gamma(\alpha-n+2)}+\frac{(1-s)^{\alpha-n}(1-t)}{\Gamma(\alpha-n+1)}, \quad t \leq s
\end{aligned}
$$

then $G_{2}(t, s)$ is a nonincreasing function with respect to $t$. Thus, we get

$$
\begin{aligned}
& \max _{t \in[0,1]} G_{1}(t, s) \leq \frac{2(1-s)^{\alpha-n+1}}{\Gamma(\alpha-n+2)}+\frac{(1-s)^{\alpha-n}}{\Gamma(\alpha-n+1)}, \\
& \min _{t \in\left[\frac{1}{4}, \frac{3}{4}\right]} G_{1}(t, s) \geq \frac{1}{4}\left[\frac{(1-s)^{\alpha-n+1}}{\Gamma(\alpha-n+2)}+\frac{(1-s)^{\alpha-n}}{\Gamma(\alpha-n+1)}\right],
\end{aligned}
$$

and

$$
\begin{aligned}
\max _{t \in[0,1]} G_{2}(t, s) & \leq \frac{(1-s)^{\alpha-n+1}}{\Gamma(\alpha-n+2)}+\frac{(1-s)^{\alpha-n}}{\Gamma(\alpha-n+1)} \\
& <\frac{2(1-s)^{\alpha-n+1}}{\Gamma(\alpha-n+2)}+\frac{(1-s)^{\alpha-n}}{\Gamma(\alpha-n+1)}, \\
\min _{t \in\left[\frac{1}{4}, \frac{3}{4}\right]} G_{2}(t, s) & \geq \frac{1}{4}\left[\frac{(1-s)^{\alpha-n+1}}{\Gamma(\alpha-n+2)}+\frac{(1-s)^{\alpha-n}}{\Gamma(\alpha-n+1)}\right] .
\end{aligned}
$$

It follows from (2.31)-(2.34) that

$$
\max _{t \in[0,1]} G(t, s) \leq m(s)=\frac{2(1-s)^{\alpha-n+1}}{\Gamma(\alpha-n+2)}+\frac{(1-s)^{\alpha-n}}{\Gamma(\alpha-n+1)}
$$

and

$$
\min _{t \in\left[\frac{1}{4}, \frac{3}{4}\right]} G(t, s) \geq \frac{1}{4}\left[\frac{(1-s)^{\alpha-n+1}}{\Gamma(\alpha-n+2)}+\frac{(1-s)^{\alpha-n}}{\Gamma(\alpha-n+1)}\right] .
$$


Hence, if we take

$$
\varphi(s)=\frac{(1-s)^{\alpha-n+1}+(\alpha-n+1)(1-s)^{\alpha-n}}{8(1-s)^{\alpha-n+1}+4(\alpha-n+1)(1-s)^{\alpha-n}},
$$

then the property (ii) holds and it is evident that $\varphi(s) \in \mathcal{C}[(0,1),(0, \infty)]$. The proof of Lemma 2.4 is completed.

Remark 2.2 From the expression of the function $\varphi(s)$, we know that $\varphi(s)>\frac{1}{8}$.

Lemma 2.5 Let $n-1<\alpha<n$. Then $H(t, s)$ given by (2.16) has the following properties:

(i) $H(t, s) \in \mathcal{C}([0,1] \times[0,1)), H(t, s)>0, t, s \in(0,1)$.

(ii) There exist nonnegative numbers $\rho$ and $\gamma$ such that

$$
H(t, s) \leq \rho m(s), \quad \text { for } t \in[0,1], s \in(0,1)
$$

and

$$
H(t, s) \geq \gamma \varphi(s) m(s), \quad t \in\left[\frac{1}{4}, \frac{3}{4}\right], s \in(0,1),
$$

where

$$
\begin{aligned}
& \rho=1+\frac{3 \eta^{\delta}}{\psi \Gamma(\delta+1)}+\frac{2\left(\eta^{2 \delta+1}-\mu^{2 \delta+1}\right)}{\psi \Gamma(\delta+1) \Gamma(\delta+2)} \\
& \gamma=1+\frac{1}{\psi \Gamma(\delta+1)}\left(\frac{3}{2}+\frac{2 \mu^{\delta+1}}{\Gamma(\delta+2)}-\frac{2 \eta^{\delta+1}}{\Gamma(\delta+2)}\right)\left(\mu-\frac{1}{4}\right)^{\delta} .
\end{aligned}
$$

Proof From the definition of $H(t, s)$, it is easy to see that property (i) is satisfied. Now, property (ii) will be verified. From (2.16) and (2.28) for $t \in[0,1], s \in[0,1)$, we get

$$
\begin{aligned}
H(t, s) \leq & m(s)+\frac{2}{\psi}\left(1+\frac{\mu^{\delta+1}}{\Gamma(\delta+2)}\right) \frac{1}{\Gamma(\delta)} \int_{0}^{\eta}(\eta-\tau)^{\delta-1} m(s) d \tau \\
& +\frac{1}{\psi}\left(1-\frac{2 \eta^{\delta+1}}{\Gamma(\delta+2)}\right) \frac{1}{\Gamma(\delta)} \int_{0}^{\mu}(\mu-\tau)^{\delta-1} m(s) d \tau \\
= & m(s)\left[1+\frac{1}{\psi \Gamma(\delta+1)}\left[2 \eta^{\delta}\left(1+\frac{\mu^{\delta+1}}{\Gamma(\delta+2)}\right)+\mu^{\delta}\left(1-\frac{2 \eta^{\delta+1}}{\Gamma(\delta+2)}\right)\right]\right] \\
\leq & m(s)\left(1+\frac{3 \eta^{\delta}}{\psi \Gamma(\delta+1)}+\frac{2\left(\eta^{2 \delta+1}-\mu^{2 \delta+1}\right)}{\psi \Gamma(\delta+1) \Gamma(\delta+2)}\right) .
\end{aligned}
$$

On the other hand, we can derive from (2.16) and (2.29) that

$$
\begin{aligned}
H(t, s) \geq & \varphi(s) m(s)+\frac{1}{\psi}\left[\left(\frac{5}{4}+\frac{2 \mu^{\delta+1}}{\Gamma(\delta+2)}-\frac{3 \mu^{\delta}}{4 \Gamma(\delta+1)}\right) \frac{1}{\Gamma(\delta)} \int_{1 / 4}^{\eta}(\eta-\tau)^{\delta-1} G(\tau, s) d \tau\right. \\
& \left.+\left(\frac{1}{4}-\frac{2 \eta^{\delta+1}}{\Gamma(\delta+2)}+\frac{3 \eta^{\delta}}{4 \Gamma(\delta+1)}\right) \frac{1}{\Gamma(\delta)} \int_{1 / 4}^{\mu}(\mu-\tau)^{\delta-1} G(\tau, s) d \tau\right] \\
\geq & \varphi(s) m(s)\left\{1+\frac{1}{\psi \Gamma(\delta+1)}\left[\left(\frac{5}{4}+\frac{2 \mu^{\delta+1}}{\Gamma(\delta+2)}-\frac{3 \mu^{\delta}}{4 \Gamma(\delta+1)}\right)\left(\eta-\frac{1}{4}\right)^{\delta}\right.\right.
\end{aligned}
$$




$$
\begin{aligned}
& \left.\left.+\left(\frac{1}{4}-\frac{2 \eta^{\delta+1}}{\Gamma(\delta+2)}+\frac{3 \eta^{\delta}}{4 \Gamma(\delta+1)}\right)\left(\mu-\frac{1}{4}\right)^{\delta}\right]\right\} \\
\geq & \varphi(s) m(s)\left[1+\frac{1}{\psi \Gamma(\delta+1)}\left(\frac{3}{2}+\frac{2 \mu^{\delta+1}}{\Gamma(\delta+2)}-\frac{2 \eta^{\delta+1}}{\Gamma(\delta+2)}\right)\left(\mu-\frac{1}{4}\right)^{\delta}\right] .
\end{aligned}
$$

Therefore, the proof of Lemma 2.5 is completed.

Lemma 2.6 If there exists $h \in Q$ such that $y(t)=\int_{0}^{1} H(t, s) h(s) d s<+\infty$, then $y(t) \geq \theta, t \in J$, i.e., $y \in Q$.

Proof By means of Lemma 2.3 and Lemma 2.5, we have $y(t) \geq \theta, t \in J$.

Remark 2.3 Define $\sigma$ as follows:

$$
\sigma=\frac{\gamma}{8}
$$

If there exists $h \in Q$ such that $y(t)=\int_{0}^{1} H(t, s) h(s) d s<+\infty$, then by Remark 2.2 and Lemma 2.3, we get

$$
\begin{aligned}
\min _{t \in\left[\frac{1}{4}, \frac{3}{4}\right]} y(t) & =\min _{t \in\left[\frac{1}{4}, \frac{3}{4}\right]} \int_{0}^{1} H(t, s) h(s) d s \\
& \geq \sigma \int_{0}^{1} m(s) h(s) d s \\
& \geq \frac{\sigma}{\rho} \rho \int_{0}^{1} m(s) h(s) d s \\
& \geq \frac{\sigma}{\rho} \int_{0}^{1} H(s, s) h(s) d s \\
& =\frac{\sigma}{\rho} y(s), \quad s \in J .
\end{aligned}
$$

To establish the existence and nonexistence of positive solutions, we define a cone $K$ by

$$
K=\left\{y \in Q: y(t) \geq \frac{\sigma}{\rho} y(s), t \in\left[\frac{1}{4}, \frac{3}{4}\right], s \in J\right\}
$$

where $\rho$ and $\sigma$ are defined by (2.38) and (2.39), respectively.

In this paper, by means of Lemma 2.1 , we will consider the boundary value problem (2.4), (2.5). Here, we define the operator

$$
\begin{aligned}
A y(t)= & \lambda_{1} \int_{0}^{1} H(t, s) a(s) f\left(s, I^{n-2} y(s), \ldots, I^{1} y(s), y(s)\right) d s \\
& +\lambda_{2} \int_{0}^{1} H(t, s) b(s) g\left(s, I^{n-2} y(s), \ldots, I^{1} y(s), y(s),\right. \\
& \left.T\left(I^{n-2} y(s)\right), S\left(I^{n-2} y(s)\right)\right) d s,
\end{aligned}
$$

where $H(t, s)$ is given by $(2.16)$. 
Lemma 2.7 Assume that (H1)-(H3) are satisfied. Then, for any $r>0$, the operator $A: Q \cap$ $B_{r} \rightarrow Q$ is a strict set contraction, where $B_{r}=\left\{y \in C[J, E]:\|y\|_{c} \leq r\right\}$.

Proof Let $y \in Q \cap B_{r}$, then from (H1), for any $t \in[0,1]$ we obtain

$$
f_{r}(t)=\sup \left\{\left\|f\left(t, u_{1}, u_{2}, \ldots, u_{n-1}\right)\right\|:\left(u_{1}, u_{2}, \ldots, u_{n-1}\right) \in P_{r}{ }^{n-1}\right\}<+\infty
$$

and

$$
\begin{aligned}
g_{r}(t) & =\sup \left\{\left\|g\left(t, u_{1}, u_{2}, \ldots, u_{n+1}\right)\right\|:\left(u_{1}, u_{2}, \ldots, u_{n+1}\right) \in P_{r}{ }^{n-1} \times P_{k^{*} r} \times P_{h^{*} r}\right\} \\
& <+\infty
\end{aligned}
$$

Using condition (H2), we have

$$
\begin{aligned}
\|A y(t)\| \leq & \rho\left[\lambda_{1} \int_{0}^{1} m(s) a(s)\left\|f\left(s, I^{n-2} y(s), \ldots, I^{1} y(s), y(s)\right)\right\| d s\right. \\
& \left.+\lambda_{2} \int_{0}^{1} m(s) b(s)\left\|g\left(s, I^{n-2} y(s), \ldots, I^{1} y(s), y(s), T\left(I^{n-2} y(s)\right), S\left(I^{n-2} y(s)\right)\right)\right\| d s\right] \\
\leq & \rho \int_{0}^{1} m(s)\left[\lambda_{1} a(s) f_{r}(s)+\lambda_{2} b(s) g_{r}(s)\right] d s<+\infty .
\end{aligned}
$$

Thus, $A: Q \cap B_{r} \rightarrow Q$ is bounded. Next, we shall prove that $A$ is continuous. Let $y_{m}, y \in$ $Q \cap B_{r}$ with $\left\|y_{m}-y\right\|_{c} \rightarrow 0$ as $m \rightarrow \infty$. For any $t_{1}, t_{2} \in J$, we have

$$
\left\|\left(A y_{m}\right)\left(t_{1}\right)-\left(A y_{m}\right)\left(t_{2}\right)\right\| \leq \int_{0}^{1}\left|H\left(t_{1}, s\right)-H\left(t_{2}, s\right)\right|\left[\lambda_{1} a(s) f_{r}(s)+\lambda_{2} b(s) g_{r}(s)\right] d s
$$

It follows from (H1), (2.42), and (2.43) that $A y_{m}$ is equicontinuous on $J$. On the other hand, for any $t \in J$, we have $\left\|y_{m}(t)-y(t)\right\| \rightarrow 0,\left\|I^{j} y_{m}(t)-I^{j} y(t)\right\| \rightarrow 0(j=1,2, \ldots, n-2)$, $\left\|S\left(I^{n-2}\right) y_{m}(t)-S\left(I^{n-2}\right) y(t)\right\| \rightarrow 0$ and $\left\|T\left(I^{n-2}\right) y_{m}(t)-T\left(I^{n-2}\right) y(t)\right\| \rightarrow 0$, as $m \rightarrow \infty$. Thus, by using the Lebesgue dominated convergence theorem and (2.42), we have

$$
\left\|\left(A y_{m}\right)(t)-(A y)(t)\right\| \rightarrow 0 \quad \text { as } m \rightarrow \infty, t \in J
$$

so $\left(A y_{m}\right)(t)$ is relatively compact for every $t \in J$. Therefore, we deduce from the AscoliArzela theorem that $\left\{A y_{m}\right\}$ is relatively compact in $Q$. Now, we will show that $\| A y_{m}-$ $A y \|_{c} \rightarrow 0$ as $m \rightarrow \infty$. If not, then there exists $\epsilon>0$ and $\left\{y_{m_{i}}\right\} \subset\left\{y_{m}\right\}$ such that

$$
\left\|A y_{m_{i}}-A y\right\|>\epsilon \quad \text { for } i=1,2, \ldots
$$

Because $\left\{A y_{m}\right\}$ is relatively compact in $\|\cdot\|_{c}$, there exists a subsequence of $\left\{A y_{m}\right\}$ converging to some $u \in C[J, P]$. Without loss of generality, we suppose that $\left\{A y_{m_{i}}\right\}$ itself converges to $u$, which means that

$$
\left\|A y_{m_{i}}-u\right\|_{c} \rightarrow 0 \quad \text { as } i \rightarrow \infty .
$$


By (2.44) and (2.46), we get $u=A y$. This relation contradicts (2.45). Hence, $A$ is a continuous operator.

Finally, we will prove that $A: Q \cap B_{r} \rightarrow Q$ is a strict set contraction, i.e., there exists $l \in(0,1)$ such that

$$
\alpha_{C}(A V) \leq l \alpha_{C}(V), \quad V \subset Q \cap B_{r}
$$

where

$$
l=\rho \int_{0}^{1} m(s)\left(\lambda_{1} a(s) L(s)+\lambda_{2} b(s) M(s)\right) d s<1,
$$

and the functions $L(s)$ and $M(s)$ are defined in (2.2) and (2.3), respectively.

Assume that $V \subset B_{r}$ is given arbitrarily, then from the above arguments, we know that $\{A y: y \in V\}$ are uniformly bounded and equicontinuous, so by Lemma 1.4 and (H2), we have

$$
\alpha_{c}(A V)=\max _{t \in[0,1]} \alpha(A V)(t)
$$

For any $y \in V$, let

$$
\begin{aligned}
\left(A_{n} y\right)(t)= & \lambda_{1} \int_{1 / n}^{1-1 / n} H(t, s) a(s) f\left(s, I^{n-2} y(s), \ldots, I^{1} y(s), y(s)\right) d s \\
& +\lambda_{2} \int_{1 / n}^{1-1 / n} H(t, s) b(s) g\left(s, I^{n-2} y(s), \ldots, I^{1} y(s), y(s),\right. \\
& \left.T\left(I^{n-2} y(s)\right), S\left(I^{n-2} y(s)\right)\right) d s .
\end{aligned}
$$

By (2.42), it is easy to see that $\left(A_{n} y\right)(t) \rightarrow(A y)(t)$ as $n \rightarrow \infty, y \in V, t \in J$. This shows that

$$
d_{H}\left(\left(A_{n} V\right)(t),(A V)(t)\right) \rightarrow 0, \quad \text { as } n \rightarrow \infty
$$

where $d_{H}(\cdot, \cdot)$ denotes the Hausdorff metric. Hence, by (2.48) and the property of the measure of noncompactness, we have

$$
\alpha\left(\left(A_{n} V\right)(t)\right) \rightarrow \alpha((A V)(t)), \quad \text { as } n \rightarrow \infty
$$

Now, we estimate for each $\alpha\left(\left(A_{n} V\right)(t)\right)$ and $t \in J$. By means of (H3), we have

$$
\begin{aligned}
\alpha\left(\left(A_{n} V\right)(t)\right) \leq & \alpha\left(\lambda_{1} \int_{1 / n}^{1-1 / n} H(t, s) a(s) f\left(s, I^{n-2} V(s), \ldots, I^{1} V(s), V(s)\right) d s\right) \\
& +\alpha\left(\lambda _ { 2 } \int _ { 1 / n } ^ { 1 - 1 / n } H ( t , s ) b ( s ) g \left(s, I^{n-2} V(s), \ldots, I^{1} V(s),\right.\right. \\
& \left.\left.V(s), T\left(I^{n-2} V(s)\right), S\left(I^{n-2} V(s)\right)\right) d s\right) \\
\leq & \lambda_{1} \rho \int_{1 / n}^{1-1 / n} m(s) a(s) \alpha\left(f\left(s, I^{n-2} V(s), \ldots, I^{1} V(s), V(s)\right)\right) d s
\end{aligned}
$$




$$
\begin{aligned}
& +\lambda_{2} \rho \int_{1 / n}^{1-1 / n} m(s) b(s) \alpha\left(g \left(s, I^{n-2} V(s), \ldots, I^{1} V(s),\right.\right. \\
& \left.\left.V(s), T\left(I^{n-2} V(s)\right), S\left(I^{n-2} V(s)\right)\right)\right) d s \\
\leq & \rho \int_{1 / n}^{1-1 / n} m(s)\left[\lambda_{1} a(s)\left(\sum_{k=1}^{n-2} \frac{L_{k}(s)}{(n-2-k) !}+L_{n-1}(s)\right)\right. \\
& +\lambda_{2} b(s)\left(\sum_{k=1}^{n-2} \frac{M_{k}(s)}{(n-2-k) !}+M_{n-1}(s)+\frac{k^{*}}{(n-3) !} M_{n}(s)\right. \\
& \left.\left.+\frac{h^{*}}{(n-3) !} M_{n+1}(s)\right)\right] d s \alpha_{c}(V) \\
\leq & \rho \int_{0}^{1} m(s)\left(\lambda_{1} a(s) L(s)+\lambda_{2} b(s) M(s)\right) d s \alpha_{c}(V),
\end{aligned}
$$

where $L(s)$ and $M(s)$ are given by (2.2) and (2.3), respectively. By using (2.49) and (2.50), we have

$$
\alpha((A V)(t)) \leq \rho \int_{0}^{1} m(s)\left(\lambda_{1} a(s) L(s)+\lambda_{2} b(s) M(s)\right) d s \alpha_{c}(V),
$$

so, by Lemma 1.4, we can get

$$
\alpha_{c}(A V) \leq \rho \int_{0}^{1} m(s)\left(\lambda_{1} a(s) L(s)+\lambda_{2} b(s) M(s)\right) d s \alpha_{c}(V),
$$

where

$$
l=\rho \int_{0}^{1} m(s)\left(\lambda_{1} a(s) L(s)+\lambda_{2} b(s) M(s)\right) d s<1 .
$$

Thus, the operator $A: Q \cap B_{r} \rightarrow Q$ is a strict set contraction. The proof of Lemma 2.7 is completed.

Lemma 2.8 Assume that (H1)-(H3) are satisfied. Then $A(K) \subset K$ and $A: K_{r, R} \rightarrow K$ is a strict set contraction.

Proof By Remark 2.3, it is obvious that the operator $A$ leaves the cone $K$ invariant; i.e., $A$ : $K \rightarrow K$. Besides, by $K_{r, R} \subset K, A\left(K_{r, R}\right) \subset K$ holds. Thus, $A: K_{r, R} \rightarrow K$. This and Lemma 2.7 complete the proof of Lemma 2.8 .

\section{Main results}

In this section, we give the existence, multiplicity, and nonexistence results of positive solutions for the BVP (1.1), (1.2).

For convenience, let us define

$$
\begin{aligned}
& A=\frac{1}{\rho}\left[\sum_{i=0}^{n-2} \frac{1}{i !} \int_{0}^{1} m(s)(a(s)+b(s)) d s+\frac{k^{*}+h^{*}}{(n-3) !} \int_{0}^{1} m(s) b(s) d s\right]^{-1}, \\
& B=\left[\int_{1 / 4}^{3 / 4} m(s) a(s) d s\right]^{-1}, \quad C=\left[\int_{1 / 4}^{3 / 4} m(s) b(s) d s\right]^{-1} .
\end{aligned}
$$


Now, we assume the following condition on $f\left(t, y_{1}, \ldots, y_{n-1}\right)$ and $g\left(t, y_{1}, \ldots, y_{n+1}\right)$.

(H4) There exist constants $0<r<R<+\infty$ such that for all $t \in J$

$$
\begin{aligned}
& \left\|f\left(t, y_{1}, \ldots, y_{n-1}\right)\right\| \leq \frac{A}{\lambda_{1}} \sum_{i=1}^{n-1}\left\|y_{i}\right\|, \quad y_{i} \in P(i=1,2, \ldots, n-1), 0 \leq \sum_{i=1}^{n-1}\left\|y_{i}\right\| \leq r, \\
& \left\|g\left(t, y_{1}, \ldots, y_{n+1}\right)\right\| \leq \frac{A}{\lambda_{2}} \sum_{i=1}^{n+1}\left\|y_{i}\right\|, \quad y_{i} \in P(i=1,2, \ldots, n+1), 0 \leq \sum_{i=1}^{n+1}\left\|y_{i}\right\| \leq r, \\
& \psi\left(f\left(t, y_{1}, \ldots, y_{n-1}\right)\right) \geq \frac{\rho R B}{\lambda_{1} \sigma^{2}}, \quad y_{i} \in P \backslash \theta(i=1,2, \ldots, n-1), R \leq \sum_{i=1}^{n-1}\left\|y_{i}\right\|<+\infty,
\end{aligned}
$$

where $\rho$ and $\sigma$ are given by (2.38) and (2.39), respectively, and $\psi \in P^{*}$ with $\|\psi\|=1$.

Theorem 3.1 Suppose that $(\mathrm{H} 1)-(\mathrm{H} 4)$ are satisfied and $P$ is normal. Then the BVP (1.1), (1.2) has at least one positive solution $y(t), t \in J$ such that

$$
\frac{\sigma}{\rho}\left(\frac{k^{*}+h^{*}}{(n-3) !}+\sum_{i=0}^{n-2} \frac{1}{i !}\right)^{-1} r \leq\|y(t)\| \leq \frac{\rho}{\sigma} R
$$

Proof Assume that the operator given by (2.41) is the cone preserving, strict set contraction. Choose

$$
r_{1}=\left(\frac{k^{*}+h^{*}}{(n-3) !}+\sum_{i=0}^{n-2} \frac{1}{i !}\right)^{-1} r
$$

It is evident that $r_{1}<r$. Let $y \in K$ with $\|y\|_{c}=r_{1}$, then by (H4), we have

$$
\begin{aligned}
\|A y(t)\| \leq & \rho\left[\lambda_{1} \int_{0}^{1} m(s) a(s)\left\|f\left(s, I^{n-2} y(s), \ldots, I^{1} y(s), y(s)\right)\right\| d s\right. \\
& \left.+\lambda_{2} \int_{0}^{1} m(s) b(s)\left\|g\left(s, I^{n-2} y(s), \ldots, I^{1} y(s), y(s), T\left(I^{n-2} y(s)\right), S\left(I^{n-2} y(s)\right)\right)\right\| d s\right] \\
\leq & \rho A\left[\int_{0}^{1} m(s)(a(s)+b(s))\left[\sum_{i=1}^{n-2}\left\|I^{i} y(s)\right\|+\|y(s)\|\right] d s\right. \\
& \left.+\int_{0}^{1} m(s) b(s)\left[\left\|T\left(I^{n-2} y(s)\right)\right\|+\left\|S\left(I^{n-2} y(s)\right)\right\|\right] d s\right] \\
\leq & \rho A\|y\|_{c}\left[\sum_{i=0}^{n-2} \frac{1}{i !} \int_{0}^{1} m(s)(a(s)+b(s)) d s+\frac{k^{*}+h^{*}}{(n-3) !} \int_{0}^{1} m(s) b(s) d s\right] \\
= & \|y\|_{c} .
\end{aligned}
$$

If we choose $\Omega_{1}=\left\{y \in K:\|y\|_{c}<r_{1}\right\}$, then we have 
Next, set $\bar{R}=\frac{\rho}{\sigma} R$ and $\Omega_{2}=\left\{y \in K:\|y\|_{c}<\bar{R}\right\}$. Then $y \in K$ with $\|y\|_{c}=\bar{R}, t \in\left[\frac{1}{4}, \frac{3}{4}\right], s \in$ $[0,1]$ implies that

$$
y(t) \geq \frac{\sigma}{\rho} y(s)
$$

so we have

$$
\|y(t)\| \geq \frac{\sigma}{\rho} \bar{R}=R, \quad t \in\left[\frac{1}{4}, \frac{3}{4}\right]
$$

that is, $\left\|\sum_{i=1}^{n-2} I^{i} y(s)\right\|+\|y(s)\| \geq R$ for all $s \in\left[\frac{1}{4}, \frac{3}{4}\right]$. Then, from condition (H4) again, we have

$$
\begin{aligned}
\|A y(t)\| & \geq \psi(A y)(t) \geq \lambda_{1} \int_{0}^{1} H(t, s) a(s) \psi\left(f\left(s, I^{n-2} y(s), \ldots, I^{1} y(s), y(s)\right)\right) d s \\
& \geq \lambda_{1} \sigma \int_{1 / 4}^{3 / 4} m(s) a(s) \psi\left(f\left(s, I^{n-2} y(s), \ldots, I^{1} y(s), y(s)\right)\right) d s \\
& \geq \frac{\rho R B}{\sigma} \int_{1 / 4}^{3 / 4} m(s) a(s) d s \\
& =\frac{\rho}{\sigma} R=\bar{R}=\|y\|_{c} .
\end{aligned}
$$

Thus, we have

$$
\|A y\|_{c} \geq\|y\|_{c} \quad \text { for all } y \in K \cap \partial \Omega_{2}
$$

Lemma 1.5 together with (3.4) and (3.5) shows that there exists a fixed point $y(t)$ in $K \cap$ $\left(\bar{\Omega}_{2} \backslash \Omega_{1}\right)$ satisfying $\frac{\sigma}{\rho}\left(\frac{k^{*}+h^{*}}{(n-3) !}+\sum_{i=0}^{n-2} \frac{1}{i !}\right)^{-1} r \leq\|y(t)\| \leq \frac{\rho}{\sigma} R$. This and Lemma 2.1 complete the proof of Theorem 3.1.

Similarly, we can prove the following result.

Corollary 3.1 Suppose that (H1)-(H3) hold and P is normal. If $f^{0}=0, g^{0}=0$ and $(\psi f)_{\infty}=$ $\infty$, then the BVP (1.1), (1.2) has at least one positive solution $y(t), t \in J$ in P for $r>0$ sufficiently small and $R>0$ sufficiently large.

In the next theorem, we also assume the following condition on $f\left(t, y_{1}, \ldots, y_{n-1}\right)$ and $g\left(t, y_{1}, \ldots, y_{n+1}\right)$.

(H5) There exist constants $0<r<R<+\infty$ such that for all $t \in J$

$$
\begin{aligned}
& \psi\left(f\left(t, y_{1}, \ldots, y_{n-1}\right)\right) \geq \frac{\rho B \sum_{i=1}^{n-1}\left\|y_{i}\right\|}{\lambda_{1} \sigma^{2}}, \quad y_{i} \in P \backslash \theta(i=1,2, \ldots, n-1), 0 \leq \sum_{i=1}^{n-1}\left\|y_{i}\right\| \leq r, \\
& \left\|f\left(t, y_{1}, \ldots, y_{n-1}\right)\right\| \leq \frac{A}{\lambda_{1}} \sum_{i=1}^{n-1}\left\|y_{i}\right\|, \quad y_{i} \in P(i=1,2, \ldots, n-1), R \leq \sum_{i=1}^{n-1}\left\|y_{i}\right\|<\infty, \\
& \left\|g\left(t, y_{1}, \ldots, y_{n+1}\right)\right\| \leq \frac{A}{\lambda_{2}} \sum_{i=1}^{n+1}\left\|y_{i}\right\|, \quad y_{i} \in P(i=1,2, \ldots, n+1), R \leq \sum_{i=1}^{n+1}\left\|y_{i}\right\|<\infty,
\end{aligned}
$$

where $\rho$ and $\sigma$ are given by (2.38) and (2.39), respectively, and $\psi \in P^{*}$ with $\|\psi\|=1$. 
Theorem 3.2 Suppose that $(\mathrm{H} 1)-(\mathrm{H} 3)$ and $(\mathrm{H} 5)$ are satisfied and $P$ is normal. Then the $B V P(1.1),(1.2)$ has at least one positive solution $y(t), t \in J$, such that

$$
\frac{\sigma}{\rho}\left(\sum_{i=0}^{n-2} \frac{1}{i !}\right)^{-1} r \leq\|y(t)\| \leq R .
$$

Proof Assume that the operator given by (2.41) is a cone preserving, strict set contraction. Choose

$$
r_{2}=\left(\sum_{i=0}^{n-2} \frac{1}{i !}\right)^{-1} r
$$

Clearly, $r_{2}<r$. Let $y \in K$ with $\|y\|_{C}=r_{2}$, then by (H5), we have

$$
\begin{aligned}
\|A y(t)\| & \geq \psi((A y)(t)) \geq \lambda_{1} \int_{0}^{1} H(t, s) a(s) \psi\left(f\left(s, I^{n-2} y(s), \ldots, I^{1} y(s), y(s)\right)\right) d s \\
& \geq \lambda_{1} \sigma \int_{1 / 4}^{3 / 4} m(s) a(s) \psi\left(f\left(s, I^{n-2} y(s), \ldots, I^{1} y(s), y(s)\right)\right) d s \\
& \geq \frac{B \rho}{\sigma} \int_{1 / 4}^{3 / 4} m(s) a(s)\left[\sum_{i=1}^{n-2}\left\|I^{i} y(s)\right\|+\|y(s)\|\right] d s \\
& \geq \frac{B \rho}{\sigma} \frac{\sigma}{\rho}\|y\|_{c} \int_{1 / 4}^{3 / 4} m(s) a(s) d s \\
& =\|y\|_{c} .
\end{aligned}
$$

Set $\Omega_{1}=\left\{y \in K:\|y\|_{c}<r_{2}\right\}$, thus we have

$$
\|A y\|_{c} \geq\|y\|_{c} \quad \text { for all } y \in K \cap \partial \Omega_{1} .
$$

Finally, let $y \in K$ with $\|y\|_{c}=R$. Then from condition (H5), we get

$$
\begin{aligned}
\|A y(t)\| \leq & \rho\left[\lambda_{1} \int_{0}^{1} m(s) a(s)\left\|f\left(s, I^{n-2} y(s), \ldots, I^{1} y(s), y(s)\right)\right\| d s\right. \\
& \left.+\lambda_{2} \int_{0}^{1} m(s) b(s)\left\|g\left(s, I^{n-2} y(s), \ldots, I^{1} y(s), y(s), T\left(I^{n-2} y(s)\right), S\left(I^{n-2} y(s)\right)\right)\right\| d s\right] \\
\leq & \rho A\left[\int_{0}^{1} m(s)(a(s)+b(s))\left[\sum_{i=1}^{n-2}\left\|I^{i} y(s)\right\|+\|y(s)\|\right] d s\right. \\
& \left.+\int_{0}^{1} m(s) b(s)\left[\left\|T\left(I^{n-2} y(s)\right)\right\|+\left\|S\left(I^{n-2} y(s)\right)\right\|\right] d s\right] \\
\leq & \rho A\|y\|_{c}\left[\sum_{i=0}^{n-2} \frac{1}{i !} \int_{0}^{1} m(s)(a(s)+b(s)) d s+\frac{k^{*}+h^{*}}{(n-3) !} \int_{0}^{1} m(s) b(s) d s\right] \\
= & \|y\|_{c} .
\end{aligned}
$$


Therefore, we have

$$
\|A y\|_{c} \leq\|y\|_{c} \quad \text { for all } y \in K \cap \partial \Omega_{2}
$$

where $\Omega_{2}=\left\{y \in K:\|y\|_{c}<R\right\}$. Lemma 1.5 together with (3.8) and (3.9) shows that there exists a fixed point $y(t)$ in $K \cap\left(\bar{\Omega}_{2} \backslash \Omega_{1}\right)$ satisfying $\frac{\sigma}{\rho}\left(\sum_{i=0}^{n-2} \frac{1}{i !}\right)^{-1} r \leq\|y(t)\| \leq R$. This and Lemma 2.1 complete the proof of Theorem 3.2.

Similarly, we can also prove the following results.

Corollary 3.2 Suppose that (H1)-(H3) hold and $P$ is normal. Iff ${ }^{\infty}=0, g^{\infty}=0$ and $(\psi f)_{0}=$ $\infty$, then the BVP(1.1)-(1.2) has at least one positive solution y in P for $r>0$ sufficiently small and $R>0$ sufficiently large.

Theorem 3.3 Suppose that (H1)-(H3) are satisfied, $P$ is normal and the following two conditions hold:

(H6) There exist constants $0<r<R<+\infty$ such that for all $t \in J$

$$
\begin{aligned}
& \psi\left(f\left(t, y_{1}, \ldots, y_{n-1}\right)\right) \geq \frac{\rho B}{\lambda_{1} \sigma^{2}} \sum_{i=1}^{n-1}\left\|y_{i}\right\|, \quad y_{i} \in P(i=1,2, \ldots, n-1), \sum_{i=1}^{n-1}\left\|y_{i}\right\| \leq r, \\
& \psi\left(g\left(t, y_{1}, \ldots, y_{n+1}\right)\right) \geq \frac{\rho C \sum_{i=1}^{n+1}\left\|y_{i}\right\|}{\lambda_{2} \sigma^{2}}, \quad y_{i} \in P(i=1,2, \ldots, n+1), \sum_{i=1}^{n+1}\left\|y_{i}\right\| \geq R .
\end{aligned}
$$

(H7) There exists $b>0$ such that

$$
\begin{aligned}
& \sup _{\left(t, y_{1}, \ldots, y_{n-1}\right) \in J \times P_{b}^{n-1}}\left\|f\left(t, y_{1}, \ldots, y_{n-1}\right)\right\|<\frac{b}{2 \lambda_{1} \rho \int_{0}^{1} m(s) a(s) d s}, \\
& \sup _{\left(t, y_{1}, \ldots, y_{n-1}\right) \in J \times P_{b}^{n-1} \times P_{k^{*} b} \times P_{h^{*} b}}\left\|g\left(t, y_{1}, \ldots, y_{n+1}\right)\right\|<\frac{b}{2 \lambda_{2} \rho \int_{0}^{1} m(s) b(s) d s},
\end{aligned}
$$

where $\rho, \sigma, k^{*}$, and $h^{*}$ are given by (2.38), (2.39), and (2.1), respectively, and $\psi \in P^{*}$ with $\|\psi\|=1$.

Then the BVP (1.1), (1.2) has at least two positive solutions.

Proof Suppose that the operator given by (2.41) is a cone preserving, strict set contraction. Let

$$
r_{3}=\left(\sum_{i=0}^{n-2} \frac{1}{i !}\right)^{-1} r
$$

Clearly, $r_{3}<r$. Then for $t \in J, y \in K$ with $\|y\|_{c}=r_{3}$, we have

$$
\begin{aligned}
\|A y(t)\| & \geq \psi((A y)(t)) \geq \lambda_{1} \int_{0}^{1} H(t, s) a(s) \psi\left(f\left(s, I^{n-2} y(s), \ldots, I^{1} y(s), y(s)\right)\right) d s \\
& \geq \lambda_{1} \sigma \int_{1 / 4}^{3 / 4} m(s) a(s) \psi\left(f\left(s, I^{n-2} y(s), \ldots, I^{1} y(s), y(s)\right)\right) d s
\end{aligned}
$$




$$
\begin{aligned}
& \geq \frac{B \rho}{\sigma} \int_{1 / 4}^{3 / 4} m(s) a(s)\left[\sum_{i=1}^{n-2}\left\|I^{i} y(s)\right\|+\|y(s)\|\right] d s \\
& \geq \frac{B \rho}{\sigma} \frac{\sigma}{\rho}\|y\|_{c} \int_{1 / 4}^{3 / 4} m(s) a(s) d s \\
& =\|y\|_{c} .
\end{aligned}
$$

If we choose $\Omega_{1}=\left\{y \in K:\|y\|_{c}<r_{3}\right\}$, then we have

$$
\|A y\|_{c} \geq\|y\|_{c} \quad \text { for all } y \in K \cap \partial \Omega_{1} .
$$

Further, set $r_{4}=\max \left\{2 r_{3}, \frac{\rho}{\sigma} R\right\}$ and $\Omega_{2}=\left\{y \in K:\|y\|_{c}<r_{4}\right\}$. Then $y \in K$ with $\|y\|_{c}=r_{4}$, $t \in\left[\frac{1}{4}, \frac{3}{4}\right], s \in[0,1]$ implies that

$$
y(t) \geq \frac{\sigma}{\rho} y(s)
$$

so we have

$$
\|y(t)\| \geq \frac{\sigma}{\rho} r_{4}=R, \quad t \in\left[\frac{1}{4}, \frac{3}{4}\right]
$$

that is, $\left\|T\left(I^{n-2} y(s)\right)\right\|+\left\|S\left(I^{n-2} y(s)\right)\right\|+\left\|\sum_{i=1}^{n-2} I^{i} y(s)\right\|+\|y(s)\| \geq R$ for all $s \in\left[\frac{1}{4}, \frac{3}{4}\right]$. Then, from condition (H6) again, we have

$$
\begin{aligned}
& \|A y(t)\| \\
& \quad \geq \psi((A y)(t)) \\
& \quad \geq \lambda_{2} \int_{0}^{1} H(t, s) b(s) \psi\left(g\left(s, I^{n-2} y(s), \ldots, I^{1} y(s), y(s), T\left(I^{n-2} y(s)\right), S\left(I^{n-2} y(s)\right)\right)\right) d s \\
& \quad \geq \lambda_{2} \sigma \int_{1 / 4}^{3 / 4} m(s) b(s) \psi\left(g\left(s, I^{n-2} y(s), \ldots, I^{1} y(s), y(s), T\left(I^{n-2} y(s)\right), S\left(I^{n-2} y(s)\right)\right)\right) d s \\
& \quad \geq \frac{\rho C}{\sigma} \int_{1 / 4}^{3 / 4} m(s) b(s)\left[\left\|T\left(I^{n-2} y(s)\right)\right\|+\left\|S\left(I^{n-2} y(s)\right)\right\|+\left\|\sum_{i=1}^{n-2} I^{i} y(s)\right\|+\|y(s)\|\right] d s \\
& \quad \geq \frac{\rho C}{\sigma} \frac{\sigma}{\rho}\|y\|_{c} \int_{1 / 4}^{3 / 4} m(s) b(s) d s \\
& \quad=\|y\|_{c} .
\end{aligned}
$$

Hence, we have

$$
\|A y\|_{c} \geq\|y\|_{c} \quad \text { for all } y \in K \cap \partial \Omega_{2}
$$

Finally, let $b \in K$ with $\|y\|_{c}=b, r_{3}<b<r_{4}$, then we get

$$
\begin{aligned}
& \|A y(t)\| \\
& \quad \leq \rho\left[\lambda_{1} \int_{0}^{1} m(s) a(s)\left\|f\left(s, I^{n-2} y(s), \ldots, I^{1} y(s), y(s)\right)\right\| d s\right.
\end{aligned}
$$




$$
\begin{aligned}
& \left.+\lambda_{2} \int_{0}^{1} m(s) b(s)\left\|g\left(s, I^{n-2} y(s), \ldots, I^{1} y(s), y(s), T\left(I^{n-2} y(s)\right), S\left(I^{n-2} y(s)\right)\right)\right\| d s\right] \\
\leq & \rho\left[\lambda_{1} \sup _{\left(t, y_{1}, \ldots, y_{n-1}\right) \in J \times P_{b}^{n-1}}\left\|f\left(t, y_{1}, \ldots, y_{n-1}\right)\right\| \int_{0}^{1} m(s) a(s) d s\right. \\
& \left.+\lambda_{2} \sup _{\left(t, y_{1}, \ldots, y_{n-1}\right) \in J \times P_{b}^{n-1} \times P_{k^{*} b} \times P_{h^{*} b}}\left\|g\left(t, y_{1}, \ldots, y_{n+1}\right)\right\| \int_{0}^{1} m(s) b(s) d s\right] \\
< & b .
\end{aligned}
$$

Therefore, we have

$$
\|A y\|_{c}<\|y\|_{c} \quad \text { for all } y \in K \cap \partial \Omega_{3}
$$

where $\Omega_{3}=\left\{y \in K:\|y\|_{c}<b\right\}$. Lemma 1.5 together with (3.11), (3.12), and (3.13) shows that there exist a fixed point $y_{1}(t)$ in $\bar{K}_{r_{3}, b}$ and a fixed point $y_{2}(t)$ in $\bar{K}_{b, r_{4}}$. This and Lemma 2.1 complete the proof of Theorem 3.3.

Now, we shall present the nonexistence results of positive solutions for the BVP (1.1), (1.2).

Theorem 3.4 Suppose that (H1)-(H3) hold, $P$ is normal and

(i) $\quad \lambda_{1} \psi\left(f\left(t, y_{1}, y_{2}, \ldots, y_{n-1}\right)\right)>\frac{\rho B}{\sigma^{2}} \sum_{i=1}^{n-1}\left\|y_{i}\right\|, \quad \forall y_{i} \in P, \sum_{i=1}^{n-1}\left\|y_{i}\right\|>0$,

or

(ii) $\lambda_{2} \psi\left(g\left(t, y_{1}, y_{2}, \ldots, y_{n+1}\right)\right)>\frac{\rho C}{\sigma^{2}} \sum_{i=1}^{n+1}\left\|y_{i}\right\|, \quad \forall y_{i} \in P, \sum_{i=1}^{n+1}\left\|y_{i}\right\|>0$,

then the BVP (1.1)-(1.2) has no positive solution.

Proof Suppose that $y(t)$ is a positive solution of the BVP (1.1)-(1.2). Then $y \in K,\|y\|_{c}>0$ for $t \in J$ and

$$
\begin{aligned}
\|y\|_{c} & \geq \sigma \lambda_{1} \int_{1 / 4}^{3 / 4} m(s) a(s) \psi\left(f\left(s, I^{n-2} y(s), \ldots, I^{1} y(s), y(s)\right)\right) d s \\
& >\frac{\rho}{\sigma} B \int_{1 / 4}^{3 / 4} m(s) a(s)\left[\sum_{i=1}^{n-2}\left\|I^{i} y(s)\right\|+\|y(s)\|\right] d s \\
& \geq \frac{\rho}{\sigma} \frac{\sigma}{\rho}\|y\|_{c} B \int_{1 / 4}^{3 / 4} m(s) a(s) d s \\
& =\|y\|_{c}
\end{aligned}
$$

which is a contradiction. Similarly, when (ii) holds, one can prove that the conclusion of Theorem 3.4 also is satisfied. This and Lemma 2.1 complete the proof. 
Theorem 3.5 Suppose that (H1)-(H3) hold, P is normal,

$$
\left\|f\left(t, y_{1}, y_{2}, \ldots, y_{n-1}\right)\right\|<\frac{A}{\lambda_{1}} \sum_{i=1}^{n-1}\left\|y_{i}\right\|, \quad \forall y_{i} \in P(i=1,2, \ldots, n-1), \sum_{i=1}^{n-1}\left\|y_{i}\right\|>0,
$$

and

$$
\left\|g\left(t, y_{1}, y_{2}, \ldots, y_{n+1}\right)\right\|<\frac{A}{\lambda_{2}} \sum_{i=1}^{n+1}\left\|y_{i}\right\|, \quad \forall y_{i} \in P(i=1,2, \ldots, n+1), \sum_{i=1}^{n+1}\left\|y_{i}\right\|>0
$$

then the BVP (1.1)-(1.2) has no positive solution.

Proof Suppose to the contrary that $y(t)$ is a positive solution of the BVP (1.1)-(1.2). Then $y \in K,\|y\|_{c}>0$ for $t \in J$, and

$$
\begin{aligned}
\|y\|_{c}= & \sup _{t \in[0,1]}\|y(t)\| \\
\leq & \rho\left[\lambda_{1} \int_{0}^{1} m(s) a(s)\left\|f\left(s, I^{n-2} y(s), \ldots, I^{1} y(s), y(s)\right)\right\| d s\right. \\
& \left.+\lambda_{2} \int_{0}^{1} m(s) b(s)\left\|g\left(s, I^{n-2} y(s), \ldots, I^{1} y(s), y(s), T\left(I^{n-2} y(s)\right), S\left(I^{n-2} y(s)\right)\right)\right\| d s\right] \\
< & \rho A\left[\int_{0}^{1} m(s)(a(s)+b(s))\left[\sum_{i=1}^{n-2}\left\|I^{i} y(s)\right\|+\|y(s)\|\right] d s\right. \\
& \left.+\int_{0}^{1} m(s) b(s)\left[\left\|T\left(I^{n-2} y(s)\right)\right\|+\left\|S\left(I^{n-2} y(s)\right)\right\|\right] d s\right] \\
\leq & \rho A\|y\|_{c}\left[\sum_{i=0}^{n-2} \frac{1}{i !} \int_{0}^{1} m(s)(a(s)+b(s)) d s+\frac{k^{*}+h^{*}}{(n-3) !} \int_{0}^{1} m(s) b(s) d s\right] \\
= & \|y\|_{c},
\end{aligned}
$$

which is a contradiction. This and Lemma 2.1 complete the proof.

\section{An example}

To demonstrate how our main results can be used in the application of our results, we give an example.

Example 4.1 Consider the following fractional boundary value problem of a finite system of scalar fractional differential equations:

$$
\begin{aligned}
{ }^{c} D^{7 / 2} x_{n}(t)= & \lambda_{1} \frac{2 e^{-t}}{\sqrt{t}}\left(x_{n}+x_{n}^{\prime}+x_{n}^{\prime \prime}\right)^{3}+\lambda_{2} \frac{e^{-2 t}}{(1-t)^{1 / 3}}\left(x_{n}+x_{n}^{\prime}+x_{n}^{\prime \prime}\right. \\
& \left.+\int_{0}^{t} \sin (t+s) e^{-s} x_{n}(s) d s+\int_{0}^{1} \cos (t-s) e^{-s} x_{n}(s) d s\right)^{2}, \\
& t \in(0,1), n=(1,2, \ldots, m),
\end{aligned}
$$




$$
\begin{aligned}
& x_{n}(0)=x_{n}^{\prime}(0)=0, \\
& x_{n}^{\prime \prime}(0)+x_{n}^{\prime \prime \prime}(0)=\frac{8}{105 \sqrt{\pi}} \int_{0}^{1 / 2}\left(\frac{1}{2}-s\right)^{7 / 2} x_{n}^{\prime \prime}(s) d s, \\
& x_{n}^{\prime \prime}(1)+x_{n}^{\prime \prime \prime}(1)+\frac{8}{105 \sqrt{\pi}} \int_{0}^{1 / 3}\left(\frac{1}{3}-s\right)^{7 / 2} x_{n}^{\prime \prime}(s) d s=0 .
\end{aligned}
$$

Conclusion System (4.1), (4.2) has at least one positive solution.

Proof Let the Banach space

$$
E=\mathbb{R}^{m}=\left\{x=\left(x_{1}, x_{2}, \ldots, x_{m}\right): x_{n} \in \mathbb{R}, n=1,2, \ldots, m\right\}
$$

with the norm $\|x\|=\max _{1 \leq n \leq m}\left|x_{n}\right|$ and $P=\left\{x=\left(x_{1}, x_{2}, \ldots, x_{m}\right): x_{n} \geq 0, n=1,2, \ldots, m\right\}$. Then $P$ is a normal cone in $E$ and problem (4.1), (4.2) can be taken into consideration as a BVP form (1.1), (1.2) in $E$. Here, $\lambda_{1}, \lambda_{2}>0$ are real numbers, $\alpha=\frac{7}{2}, \delta=\frac{9}{2}, \eta=\frac{1}{2}, \mu=\frac{1}{3}$, $a(t)=\frac{1}{\sqrt{t}}, b(t)=\frac{1}{(1-t)^{1 / 3}}, K(t, s)=\sin (t+s) e^{-s}, H(t, s)=\cos (t-s) e^{-s}, x=\left(x_{1}, x_{2}, \ldots, x_{m}\right), f=$ $\left(f_{1}, f_{2}, \ldots, f_{m}\right), g=\left(g_{1}, g_{2}, \ldots, g_{m}\right)$, where

$$
\begin{aligned}
& f_{n}(t, u, v, w)=2 e^{-t}\left(u_{n}+v_{n}+w_{n}\right)^{3}, \\
& g_{n}(t, u, v, w, z, \tau)=e^{-2 t}\left(u_{n}+v_{n}+w_{n}+z_{n}+\tau_{n}\right)^{2} .
\end{aligned}
$$

Clearly, $f \in \mathcal{C}\left[J \times P^{3}, P\right], g \in \mathcal{C}\left[J \times P^{5}, P\right](J=[0,1])$, and $P^{*}=P$, thus we can choose $\psi=$ $(1,1, \ldots, 1)$; then for any $x \in P$ we get

$$
\psi(f(t, u, v, w))=\sum_{n=1}^{m} f_{n}(t, u, v, w)
$$

Now, conditions (H1)-(H3) will be verified. It is easy to see that (H1) holds. Observe that, for any $t \in(0,1)$ and $r>0$, we have

$$
f_{r}(t) \leq 54 e^{-t} r, \quad g_{r}(t) \leq 25 e^{-2 t} r .
$$

Thus (H2) is satisfied. Furthermore, assumption (H3) is satisfied automatically since $E$ is finite dimensional.

On the other hand,

$$
\begin{aligned}
& f^{0}=\lim _{(\|u\|+\|v\|+\|w\|+\|z\|) \rightarrow 0} \sup \max _{t \in J} \frac{\|f(t, u, v, w)\|}{\|u\|+\|v\|+\|w\|}=0, \\
& g^{0}=\lim _{(\|u\|+\|v\|+\|w\|+\|z\|+\|\tau\|) \rightarrow 0} \sup \max _{t \in J} \frac{\|g(t, u, v, w, z, \tau)\|}{\|u\|+\|v\|+\|w\|+\|z\|+\|\tau\|}=0,
\end{aligned}
$$

and

$$
\frac{\psi(f(t, u, v, w))}{\|u\|+\|v\|+\|w\|} \geq \frac{\|f(t, u, v, w)\|}{\|u\|+\|v\|+\|w\|} \rightarrow \infty \quad(\|u\|+\|v\|+\|w\| \rightarrow \infty),
$$

which means $(\psi f)_{\infty}=\infty$. Therefore, Corollary 3.1 shows that (4.1), (4.2) has a solution. 


\section{Competing interests}

The author declares that she has no competing interests.

Received: 26 November 2014 Accepted: 17 February 2015 Published online: 03 March 2015

\section{References}

1. Zhao, Y, Chen, H, Huang, L: Existence of positive solutions for nonlinear fractional functional differential equation. Comput. Math. Appl. 64(10), 3456-3467 (2012)

2. Zhang, S: Positive solutions to singular boundary value problem for nonlinear fractional differential equation. Comput. Math. Appl. 59, 1300-1309 (2010)

3. Guezane Lakouda, A, Khaldi, R: Solvability of a fractional boundary value problem with fractional integral condition. Nonlinear Anal. 75, 2692-2700 (2012)

4. Kilbas, AA, Srivastava, HM, Trujillo, Jj: Theory and Applications of Fractional Differential Equations. North-Holland Mathematics Studies, vol. 204. Elsevier, Amsterdam (2006)

5. Podlubny, I: Fractional Differential Equations. Academic Press, San Diego (1999)

6. Zhang, S: Positive solutions for boundary value problems of nonlinear fractional differential equations. Electron. J. Differ. Equ. 2006, 36 (2006)

7. Zhao, Y, Yang, L, Chen, P: Positive solutions to boundary value problems of a high order fractional differential equation in a Banach space. Adv. Differ. Equ. 2013, 344 (2013)

8. Zhou, WX, Chu, YD: Existence of solutions for fractional differential equations with multi-point boundary conditions. Commun. Nonlinear Sci. Numer. Simul. 17, 1142-1148 (2012)

9. Feng, M, Zhang, X, Ge, W: New existence results for higher-order nonlinear fractional differential equation with integral boundary condition. Bound. Value Probl. 2011, 720702 (2011)

10. Zhou, J, Feng, M: Green's function for Sturm-Liouville-type boundary value problems of fractional order impulsive differential equations and its application. Bound. Value Probl. 2014, 69 (2014)

11. Agarwal, RP, O'Regan, D, Wong, PJY: Positive Solutions of Differential, Difference and Integral Equations. Kluwer Academic, Boston (1999)

12. Agarwal, RP, Wong, PJY: Existence of solutions for singular boundary value problems for higher order differential equations. Milan J. Math. 65, 249-264 (1995)

13. Hao, $X, L i u, L, W u, Y, X u, N$ : Multiple positive solutions for singular $n$ th-order nonlocal boundary value problems in Banach spaces. Comput. Math. Appl. 61, 1880-1890 (2011)

14. Wong, PJY, Agarwal, RP: Eigenvalues of boundary value problems for higher order differential equations. Math. Probl. Eng. 2, 401-434 (1996)

15. Zhang, $X$, Yang, $X, G e, W$ : Positive solutions of $n$ th-order impulsive boundary value problems with integral boundary conditions in Banach spaces. Nonlinear Anal. 71, 5930-5945 (2009)

16. Zhang, X, Feng, M, Ge, W: Existence and nonexistence of positive solutions for a class of $n$th order three-point boundary value problems in Banach spaces. Nonlinear Anal. 70, 584-597 (2009)

17. Zhang, L, Bashir, A, Wang, G, Agarwal, RP: Nonlinear fractional integro-differential equations on unbounded domains in a Banach space. J. Comput. Appl. Math. 249, 51-56 (2013)

18. Guo, D, Lakshmikantham, V, Liu, X: Nonlinear Integral Equations in Abstract Spaces. Kluwer Academic, Dordrecht (1996)

19. Guo, D, Lakshmikantham, V: Nonlinear Problems in Abstract Cones. Academic Press, New York (1988)

20. Lakshmikantham, V, Leela, S: Nonlinear Differential Equations in Abstract Spaces. Pergamon, Oxford (1981)

21. Demling, K: Ordinary Differential Equations in Banach Spaces. Springer, Berlin (1977)

\section{Submit your manuscript to a SpringerOpen ${ }^{\circ}$ journal and benefit from:}

- Convenient online submission

Rigorous peer review

- Immediate publication on acceptance

- Open access: articles freely available online

- High visibility within the field

- Retaining the copyright to your article 